\title{
Metodología para fortalecer la exposición magistral usando TIC en un curso de programación de computadores en un programa de ingeniería
}

\section{A methodology to improve the lecture classes using ICT in a programming computer course}

\author{
págs. 11-21
}

Grupo de investigación: Grupo de Investigación en Informática Línea de investigación: Desarrollo de un modelo pedagógico basado en e-Learning

Omar Iván Trejos Buriticá.

Recibido: agosto 01 de 2016

Aceptado: noviembre 25 de 2016

\begin{abstract}
In this article we examine the possible changes that occur in computer programming course when we use TIC strategies to capitalize them, all from a classroom implementation and design centered masterly exposition. The methodology used is framed in educational qualitative research with a quantitative component in conjunction with a case study. We use (simultaneous) the word processor Word, PowerPoint slides designer, the Dev-C++ development environment and the browser Google Chrome. The results show that with a simultaneous use of ICT in a course of computer programming, not only masterly exposition is strengthened notoriously but establishes more meaningful learning with more sense, strengthening independent learning and appropriation of knowledge in the long term.
\end{abstract}

Key words: ICT, computer programming, System's Engineering, masterly exposition, methodology
Palabras clave: Ingeniería de sistemas, Exposición magistral, Metodología, TIC, programación de computadores

- Ingeniero de sistemas, docente investigador, Facultad de Ingenierías, Universidad Tecnológica de Pereira. omartrejos@utp.edu.co 


\section{INTRODUCCIÓN}

Los docentes de los programas de Ingeniería han de desarrollar competencias que los sintonicen con los estudiantes de estos tiempos actuales para quienes su lenguaje natural está inmerso en el mundo de la tecnología. Esto les permitirá responder, de una manera más apropiada, a las inquietudes que, al respecto del conocimiento disciplinar, tengan los alumnos toda vez que dicha sintonía forme parte de los elementos motivacionales necesarios (Ausubel, 1963) que estén incorporados en los procesos de formación que el mismo docente lidere. En este sentido, si bien es cierto que los docentes de ingenierías tienen formación para ser Ingenieros también lo es que los docentes de ingeniería requieren tener mucha consciencia de que su labor como tales requiere conocer tanto de su campo disciplinar como Ingenieros como de su labor como docentes y por ello se necesita que tengan elementos de juicio que permitan participar, liderar y conducir procesos de aprendizaje que sean exitosos tanto para el docente como, deseablemente, para cada uno de sus estudiantes (Barriga Arceo \& Hernandez Rojas, 2002).

Debe entenderse que si bien la relación entre los docentes y las nuevas tecnologías de la información y la comunicación (TIC) no son tan activas y permanentes como la que tienen los estudiantes, se hace necesario que se fortalezca dicha relación para que entre docentes y estudiantes se establezca un puente de comunicación basado en el mismo lenguaje como es el lenguaje de la tecnología. Con la metodología que se propone en este artículo, y basado en los resultados obtenidos, se puede inferir que cuando el docente acude a las herramientas tecnológicas modernas, que le son familiares al estudiante, es mucho más fácil alcanzar los objetivos de aprendizaje que se han propuesto en cursos como los de programación de computadores y que se fortalece la comunicación entre estudiantes y docentes, promoviendo con ello el aprendizaje autónomo y la participación activa de los estudiantes en sus propios procesos y ritmos de aprendizaje.

La metodología que se expone en este artículo es producto de la interacción de su autor con estudiantes de I Semestre de Ingeniería de Sistemas y Computación de la Universidad Tecnológica de Pereira y ha sido diseñada, aplicada, refinada y retroalimentada de acuerdo a estas premisas. Tanto su forma de aplicación y conducción como los resultados obtenidos se presentan en el ítem Metodología y Resultados de este mismo artículo. Es de anotar que se ha procurado ser riguroso en aplicar la metodología informando a los alumnos permanentemente sus características y los procedimientos de medición que se han de aplicar para analizar tanto los resultados cualitativos como los cuantitativos.

Si bien no se puede pensar en que existe una forma excelsa de realizar una exposición magistral, como parte de las estrategias para que el docente presente la fundamentación que subyace a los conocimientos disciplinares necesarios (Onrubia, Febrero 2005), tampoco se puede menospreciar esta dado que sigue siendo el espacio de interacción más directo entre docentes y estudiantes. Tampoco se puede negar que, en tiempos modernos, la utilización de las TIC provee al docente de nuevas herramientas para fortalecer dicha interacción (Ministerio de las TIC, 2012) y, por lo tanto, éste ha de estar actualizado propiciando esas nuevas formas de comunicación para bien del alcance de los objetivos de aprendizaje.

Este artículo plantea, desde la óptica del docente, una forma de articular el conocimiento disciplinar, la exposición magistral, las nuevas TIC y el espectro de dudas e inquietudes que el estudiante pudiere tener de manera que, todas a una, posibiliten un entorno de aprendizaje más expedito y más cercano al lenguaje natural de los jóvenes de hoy. Se pretende con esta propuesta que la ex- 
posición magistral se dinamice a partir de herramientas tecnológicas modernas y que, de su buena utilización, se puedan derivar réditos beneficiosos para el proceso de aprendizaje. De la misma forma se busca con la metodología propuesta encontrar un camino que el afianzamiento del conocimiento previo y la solidificación del nuevo conocimiento a partir de la combinación de las TIC que posteriormente se mencionarán.

Subyace una pregunta de investigación a esta propuesta metodológica: ¿es posible encontrar un camino pedagógico que permita fortalecer la exposición magistral a partir del uso de las TIC en el aula de forma que se convierta el respaldo para el logro de los objetivos de aprendizaje? La respuesta está contenida en este artículo. No en vano la Unesco ha establecido un enfoque de competencias en TIC para los docentes en el cual deja de presente que los docentes de hoy desempeñan nuevas funciones y requieren nuevos planteamientos en su formación pues requieren integrar las TIC en el aula lo cual dependerá de la capacidad de los maestros para estructurar el ambiente de aprendizaje de forma no tradicional fusionando las TIC con nuevas pedagogías de forma que se fomenten clases dinámicas (UNESCO, 2008).

El presente artículo es un producto del proyecto de investigación sin financiación código 6-16-13 "Desarrollo de un modelo metodológico para el aprendizaje de la programación imperativa en Ingeniería de Sistemas basado en Aprendizaje Significativo, Aprendizaje por Descubrimiento y el Modelo 4Q de preferencias de pensamiento" aprobado por la Vicerrectoría de Investigación, Extensión e Innovación de la Universidad Tecnológica de Pereira.

\section{MARCO TEÓRICO}

El término "metodología" implica abstraer un concepto que gira alrededor de un conjunto de procedimientos, aceptado por una comunidad, que es usado para que se logren objetivos definidos que puedan ser descritos bien en lo cuantitativo, bien en lo cualitativo o bien en ambos y que, preferiblemente, se circunscribe dentro del ámbito de la ciencia (Lerma Gonzalez, 2014). Cuando se establecen relaciones entre un método determinado que se considere pertinente a una investigación también se puede hablar de metodología (Briones, 1996).

También resulta procedente considerar como Metodología ese grupo de métodos que han sido validados, que son aceptados y que se han comprobado para poder alcanzar objetivos dentro de un proceso investigativo. En esencia un procedimiento cualquiera, independiente y posiblemente efectivo, no es en sí mismo una metodología (Cortés \& Iglesias, 2004). Una metodología implica subprocesos de adquisición de información, análisis, decantación y validación de su pertinencia, ordenamiento y análisis así como la posibilidad de llegar a conclusiones con carácter científico que representen una aproximación a la naturaleza, sus leyes y sus formas de interacción (Hernandez Sampieri, Fernandez Collado, \& Baptista Lucio, 2006). Toda metodología tiene asociada a ella un conjunto de postulados que son su fundamento y que propenden por mejorar, ampliar, retroalimentar y ampliar el conocimiento humano sobre la realidad natural (Sabino, 2002).

La exposición o clase magistral es la estrategia mediante la cual el docente, basado en un conocimiento disciplinar pertinente y en un conjunto de habilidades, comparte con los asistentes dicho conocimiento (Hall, Waitz, Brodeur, Soderholm, \& Nasr, 2002) buscando que esto suceda de manera organizada, sistemática y que siempre propenda por lograr unos objetivos de aprendizaje específico. 
Normalmente la exposición o clase magistral sucede dentro de un aula de clases aunque no es absolutamente obligatorio que así sea.

La exposición magistral ha sido el espacio de interacción más directo que puede darse entre estudiantes y docentes dado que la comunicación se da frente a frente y eso posibilita una dinámica favorable al aprendizaje que hasta ahora no ha sido superada (Gonzalez, 2002). Debe aclararse que diferentes servicios telemáticos modernos se han aproximado a esta interacción (entiéndase como tales los servicios Line, Skype, whatsapp y otros) sin embargo aunque se han logrado aproximaciones, especialmente para aquellos estudiantes que se encuentran distantes del lugar en donde sucede la clase magistral, siempre dependerán de las condiciones técnicas que posibiliten la interacción y, contrario a la clase magistral directa, no tienen la posibilidad de que la comunicación incluya esos elementos invisibles que se dan en la interacción directa cuando ésta sucede de manera personal o, en caso de que ésta se dé, aún no se dan las condiciones para que esos elementos invisibles (contacto, calor humano, miradas directas, expresiones faciales explícitas y otras) puedan ser capitalizables a partir del uso de dichas herramientas.

Se conocen como TIC (acrónimo de Tecnologías de la Información y la Comunicación) todo el conjunto de tecnologías desarrolladas para facilitar la presentación, exposición, gestión, almacenamiento y difusión de la información de forma que pueda ser enviada de un lugar a otro por medios electrónicos y que, posteriormente, pueden ser recuperadas, revisadas, procesadas, decantadas, retroalimentadas y reenviadas de acuerdo a determinadas necesidades. En la actualidad, las TIC han ocupado un espacio muy importante en el desarrollo de las actividades que suceden en los tres contextos escolares (contexto del aula, contexto institucional y contexto extraescolar) (Palomar Sanchez, 2009) puesto que la tecnología se ha ido convirtiendo poco a poco en el lenguaje natural de los jóvenes de hoy adicional al hecho de que estas TIC posibilitan a) el acceso a las fuentes de información como nunca antes se había logrado, b) a comunicación síncrona y asíncrona en el momento en que se presentan dudas y c) la posibilidad de interactuar virtualmente como no se había podido hacer antes (Trejos Buriticá, 2013).

Cuando se habla de las TIC no solo se habla de programas (software) que cristalizan servicios sino que se habla de dispositivos que los posibilitan bien en el aula o bien fuera de ella (Salinas, 2004). La alta penetración comercial de los dispositivos considerados dentro del espectro de las TIC y de sus servicios asociados implica que el docente se vea invitado a conocerlas, aprenderlas a usar, capitalizarlas al máximo y buscar con ellas caminos que faciliten los procesos de aprendizaje tanto dentro del aula como fuera de ella.

La programación de computadores se define como el proceso a través del cual aprovechando las posibilidades y recursos de un lenguaje de programación, se implementa una solución a un problema determinado (Van Roy, 2008), de manera que los resultados pueden confrontarse posteriormente con los objetivos que se pretendían alcanzar y siempre buscando que la tecnología computacional posibilite un camino más simple para dicha implementación. La programación de computadores se realiza a través de lenguajes de programación que son conjuntos de instrucciones que, valiéndose de entornos de programación, permiten implementar soluciones ejecutables a problemas computables (Trejos Buriticá, La Esencia de la Lógica de Programación, 2000).

Un paradigma de programación es un modelo de solución que aprovecha ciertos recursos y ciertas posibilidades para encontrar, desde un enfoque determinado, una solución posible a un problema determinado que, normalmente, es computable y cuya solución es implementable en un com- 
putador (Deitel \& Deitel, 2013). El paradigma provee un enfoque y un conjunto de herramientas que hacen que sea posible buscar una solución a partir de unos recursos que, normalmente, la matemática brinda. La programación funcional, la programación imperativa y la programación orientada a objetos obedecen a paradigmas diferentes. En la programación funcional se priorizan los procesos y las formas como estos procesos interactúan (Felleisen, 2006), en la programación imperativa el concepto de variables y cambios de estado son los que le dan fundamento al paradigma (Schildt, 2010) y en la programación orientada a objetos la conjunción entre métodos y atributos hace que sea posible encontrar un modelo que interprete el mundo real (Schildt, C++ Programming, 2010).

\section{METODOLOGÍA}

La metodología sugerida en este artículo se aplicó en los cursos Programación II de Ingeniería de Sistemas y Computación de la Universidad Tecnológica de Pereira durante el $1^{\circ}$ y $2^{\circ}$ semestre del año 2015. Partiendo de que el contenido de las asignaturas esta parcelado por semanas, se escogió una semana (que está compuesta por 3 sesiones), que equivale a un tema, para realizar la exposición magistral como se hace tradicionalmente (es decir sin ningún apoyo en TIC) y el siguiente tema se expuso apoyado en la metodología basada en TIC sugerida en este artículo. Se buscó que los temas fueran similares en complejidad y aplicación y que cada tema tomara una semana completa. La tabla 1 presenta los temas y los recursos utilizados.

Tabla 1.

Temas y recursos utilizados

\begin{tabular}{lllll}
\hline \multirow{2}{*}{ Metodología } & \multirow{2}{*}{ Tema } & \multicolumn{3}{c}{ Recursos Utilizados } \\
\cline { 3 - 5 } & & \multicolumn{2}{c}{$\mathbf{1}^{\text {a } \text { Sesión }}$} & \multicolumn{2}{c}{$\mathbf{2}^{\text {a Sesión }}$} & \multicolumn{1}{c}{$\mathbf{3}^{\text {a Sesión }}$} \\
\hline \multirow{2}{*}{ Sin MS } & \multirow{2}{*}{ Ciclo for } & Tablero & Tablero & Tablero \\
& & Marcadores & Marcadores & Marcadores \\
& & VideoBeam & VideoBeam & VideoBeam \\
& & Word & Word & Word \\
Con MS & PowerPoint & IDE DevC++ & PowerPoint \\
& \multirow{4}{*}{ Ciclo while } & Navegador & Navegador & Navegador \\
& & Google & Google & Google \\
& & e-mail & whatsapp & e-mail \\
& & & & whatsapp \\
\hline
\end{tabular}

MS = Metodología Sugerida

Fuente: elaboración propia

En la tabla 2 se presentan una breve descripción de los recursos utilizados y la forma como cada recurso se ha usado dentro del contexto de la metodología propuesta. 
Tabla 2.

Descripción de recursos

\begin{tabular}{|c|c|c|}
\hline Recurso & Descripción & Utilidad \\
\hline Tablero & $\begin{array}{l}\text { Espacio físico de madera y acrílico } \\
\text { sobre el cual se puede escribir y en } \\
\text { donde se consigna lo que el docente } \\
\text { considere pertinente }\end{array}$ & $\begin{array}{l}\text { Se ha utilizado como tradicionalmente se hace. } \\
\text { En la aplicación de la metodología, el tablero } \\
\text { ha servido para proyectar el haz de luz del } \\
\text { videobeam }\end{array}$ \\
\hline Marcadores & $\begin{array}{l}\text { Corresponden al recurso que se utiliza } \\
\text { para escribir sobre el tablero de manera } \\
\text { que lo que se escriba sea claro y legible }\end{array}$ & $\begin{array}{l}\text { Se han utilizado marcadores de colores para } \\
\text { poder hacer énfasis en determinadas anotaciones } \\
\text { específicas }\end{array}$ \\
\hline VideoBeam & $\begin{array}{l}\text { Dispositivo que permite desplegar sobre } \\
\text { una superficie plana el conjunto de } \\
\text { datos, aplicaciones e información que } \\
\text { aparece en una pantalla de computador }\end{array}$ & $\begin{array}{l}\text { Se utiliza para visualizar la interacción con } \\
\text { los diferentes programas y los conceptos, } \\
\text { aplicaciones, ejecuciones y textos que sean } \\
\text { pertinentes dentro del contexto de la asignatura }\end{array}$ \\
\hline Word & $\begin{array}{l}\text { Procesador de palabra que permite } \\
\text { escribir texto y concederle algunos } \\
\text { atributos de autoedición que hacen más } \\
\text { entendible y comunicable lo escrito }\end{array}$ & $\begin{array}{l}\text { El procesador de palabra Word se ha utilizado para } \\
\text { escribir, al ritmo de la clase y durante el desarrollo de } \\
\text { ella, las reflexiones, teorías, ejemplos y acotaciones } \\
\text { que se consideran importantes y pertinentes }\end{array}$ \\
\hline PowerPoint & $\begin{array}{l}\text { Programa que permite diseñar } \\
\text { diapositivas aprovechando los recursos } \\
\text { estáticos de tipos de letra, tamaño, } \\
\text { imágenes, fondos y formas que hacen } \\
\text { más entendible su contenido }\end{array}$ & $\begin{array}{l}\text { El programa de diapositivas PowerPoint se ha } \\
\text { utilizado para construir diapositivas, durante la } \\
\text { clase, de forma que cada una de ellas contenga } \\
\text { SOLAMENTE una frase que condense un tópico } \\
\text { específico de la temática expuesta }\end{array}$ \\
\hline $\begin{array}{l}\text { Navegador } \\
\text { Google }\end{array}$ & $\begin{array}{l}\text { Programa que permite conectarse a la } \\
\text { Internet en su servicio más popular: } \\
\text { la Word Wide Web (WWW) de forma } \\
\text { que se puede acceder a sitios web con } \\
\text { información pertinente }\end{array}$ & $\begin{array}{l}\text { El navegador se ha utilizado para acceder, a } \\
\text { manera de consulta y durante la sesión, a sitios } \\
\text { web con información considerada como pertinente } \\
\text { dentro del contexto de la asignatura }\end{array}$ \\
\hline e-mail & $\begin{array}{l}\text { Servicio telemático que permite que } \\
\text { un usuario pueda enviar mensajes y } \\
\text { recibirlos en una cuenta privada a la } \\
\text { manera del correo tradicional }\end{array}$ & $\begin{array}{l}\text { Se ha utilizado para que, al finalizar la clase, se } \\
\text { le envíe a todos los estudiantes registrados los } \\
\text { documentos generados en Word y PowerPoint } \\
\text { durante la clase así como los códigos de los } \\
\text { programas que se han construido }\end{array}$ \\
\hline IDE DevC++ & $\begin{array}{l}\text { Entorno integrado de desarrollo que } \\
\text { permite escribir un programa en lenguaje } \\
\text { C o C++, revisarlo, almacenarlo, } \\
\text { compilarlo, ejecutarlo y corregirlo }\end{array}$ & $\begin{array}{l}\text { Se ha utilizado para visualizar, aprovechando las } \\
\text { bondades del videobeam, el proceso completo de } \\
\text { construcción de un programa durante la clase y al } \\
\text { mismo tiempo en que se usan los otros programas }\end{array}$ \\
\hline whatsapp & $\begin{array}{l}\text { Servicio específico para los dispositivos } \\
\text { celulares que permite el envío y } \\
\text { recepción de mensajes }\end{array}$ & $\begin{array}{l}\text { Se ha utilizado para que los estudiantes por } \\
\text { ese medio, tengan comunicación directa con } \\
\text { el docente de forma que puedan presentar sus } \\
\text { inquietudes al respecto del tema especialmente } \\
\text { cuando la clase ha terminado }\end{array}$ \\
\hline
\end{tabular}

Fuente: elaboración propia 


\section{RESULTADOS}

La tabla 3 presenta el promedio de los resultados cuantitativos obtenidos en la evaluación de los temas vistos y la tabla 4 presenta los resultados cualitativos (opiniones de algunos estudiantes) en referencia a la metodología utilizada.

Tabla 3.

Resultados Cuantitativos

\begin{tabular}{clccc}
\hline Sem & \multicolumn{1}{c}{ Metodología } & Promedio & Mín. Nota & Máx. Nota \\
\hline I & Sin MS & 3.3 & 3.0 & 3.7 \\
2015 & Con MS & 3.9 & 3.5 & 4.1 \\
\multirow{2}{*}{ II } & Sin MS & 3.4 & 3.2 & 3.5 \\
2015 & Con MS & 4.1 & 4.0 & 4.5 \\
\hline
\end{tabular}

Fuente: elaboración propia

Tabla 4.

Resultados Cualitativos*

\begin{tabular}{|c|c|c|c|c|}
\hline Sem & Metodol. & Opinión A & Opinión B & Opinión C \\
\hline \multirow{2}{*}{$\begin{array}{c}1 \\
2015\end{array}$} & Sin MS & $\begin{array}{l}\text { La clase tradicional es } \\
\text { buena, el profesor explica } \\
\text { muy bien, es ordenado y se } \\
\text { hace entender }\end{array}$ & $\begin{array}{l}\text { Muy buena distribución } \\
\text { del tablero, el uso de } \\
\text { los colores es bastante } \\
\text { interesante, el tema es muy } \\
\text { claro }\end{array}$ & $\begin{array}{l}\text { Me gustaría que al } \\
\text { momento de hacer los } \\
\text { programas pudiéramos } \\
\text { verlos en el computador } \\
\text { directamente }\end{array}$ \\
\hline & Con MS & $\begin{array}{l}\text { Maravillosa la metodología, } \\
\text { la interacción entre } \\
\text { los programas es } \\
\text { verdaderamente útil }\end{array}$ & $\begin{array}{l}\text { El uso de los programas } \\
\text { con el videobeam le } \\
\text { permite a uno entender } \\
\text { mucho mejor todo }\end{array}$ & $\begin{array}{l}\text { Ver cómo se construye un } \\
\text { programa desde cero es } \\
\text { la para que uno también } \\
\text { pueda hacerlo }\end{array}$ \\
\hline \multirow{2}{*}{$\begin{array}{c}\text { II } \\
2015\end{array}$} & Sin MS & $\begin{array}{l}\text { El profe responde bien } \\
\text { a las preguntas, se nota } \\
\text { que sabe y que tiene } \\
\text { mucha experiencia como } \\
\text { programador }\end{array}$ & $\begin{array}{l}\text { Algunas cosas que el profe } \\
\text { explica en el tablero no } \\
\text { funcionan cuando uno las } \\
\text { pasa al computador }\end{array}$ & $\begin{array}{l}\text { El profe se hace entender } \\
\text { pero uno tiene que } \\
\text { imaginarse muchas cosas } \\
\text { porque no ve directamente } \\
\text { lo que él dice }\end{array}$ \\
\hline & Con MS & $\begin{array}{l}\text { Cada programa tiene } \\
\text { su utilidad y todo gira } \\
\text { alrededor de una buena } \\
\text { explicación }\end{array}$ & $\begin{array}{l}\text { Maravillosas estas clases!!! } \\
\text { (sic) me encantan, la } \\
\text { facilidad con que se ve todo } \\
\text { es increíble }\end{array}$ & $\begin{array}{l}\text { Me parece muy bueno } \\
\text { que el profe nos pregunte } \\
\text { algunas cositas de los } \\
\text { programas. Todas las } \\
\text { clases deberían ser así }\end{array}$ \\
\hline
\end{tabular}

*Se han seleccionado solo algunas opiniones

Fuente: elaboración propia 
Finalmente la tabla 5 presenta un resumen de las opiniones de los estudiantes que han participado en este proceso investigativo.

Tabla 5.

Resumen de opiniones

\begin{tabular}{clccc}
\hline Sem & \multicolumn{1}{c}{ Metodología } & Opinión favorable & Opinión desfavorable & Total estuds \\
\hline \multirow{2}{*}{ I 2015} & Sin MS & 10 & 9 & 19 \\
& Con MS & 17 & 2 & 19 \\
\multirow{2}{*}{2015} & Sin MS & 17 & 12 & 22 \\
& Con MS & 21 & 1 & 22 \\
\hline
\end{tabular}

Fuente: elaboración propia

\section{DISCUSIÓN}

Para la aplicación de la metodología sugerida se optó por escoger dos temas cercanos en contenido y en tiempo dentro de la misma asignatura de forma que los estudiantes pudieran opinar al respecto en cuanto a su ausencia o su presencia dado que son los protagonistas de la escena académica en donde se desarrolló la investigación. Se procuró también que se establecieran las mismas condiciones académicas, en todos los sentidos, para que los estudiantes pudieran establecer un comparativo entre la clase sin la metodología y sin ella, sabiendo que se acude a temas similares $y$, conceptualmente, cercanos

La clase se fortalece con la utilización de los recursos multimediales informáticos y el uso de las TIC sin embargo no son estrictamente obligatorios dado que la clase magistral tradicional tiene también sus propias ventajas. No se puede negar, eso sí, que el uso de los nuevos recursos informáticos posibilitan mayores opciones de almacenamiento, acceso, despliegue, estética y otros factores que, de una u otra forma, inciden en el proceso de aprendizaje dado que corresponden al lenguaje y entorno natural de los jóvenes de hoy.

Debe tenerse en cuenta que se han utilizado unos programas específicos pero que no es requisito que sean éstos los que se utilicen puntualmente. En general, en vez de hablar de Word, PowerPoint, Google y whatsapp puede aceptarse que se utilice cualquier Procesador de Palabra, Diseñador de Diapositivas, Navegador y cualquier programa de interacción asíncrona de texto e imágenes por dispositivos móviles. La tabla 2 describe tanto lo que es en sí cada programa utilizado, en términos generales, como el uso que se le dio a la luz de la metodología sugerida.

Es de destacar la estética geométrica de distribución del tablero y el uso de marcadores de colores en la metodología tradicional así como la utilización del procesador de palabra para consignar toda la explicación de la exposición magistral, el uso del diseñador de diapositivas para almacenar lo clave de dicha exposición, el intenso uso del videobeam, la facilidad de acceso a las fuentes de información a través del navegador y la Word Wide Web, la versatilidad del correo electrónico y la instantaneidad del servicio de servicio de whatsapp. Igualmente debe destacarse el uso de estos recursos como complemento a un entorno integrado de desarrollo (también conocido como IDE) para la concepción, confección, codificación, compilación, ejecución y almacenamiento de los programas requeridos para ejemplificar los conceptos o temas seleccionados. 
Los resultados cuantitativos que presenta la tabla 3 reflejan una significativa diferencia favorable en los temas vistos a la luz de la metodología sugerida por encima de los resultados equivalentes en los temas que se cubrieron sin acudir a dicha metodología. Como se puede observar siempre fueron superiores los promedios de las notas cuando se utilizó la metodología sugerida en este artículo que cuando no se utilizó. La relación del promedio con las notas mínimas y máximas indica que, cuantitativamente, fueron más las notas que estuvieron por encima del promedio cuando se aplicó la metodología que cuando no se aplicó, caso en el cual fueron más las notas que estuvieron por debajo del mismo. En ambos periodos de análisis, las notas fueron superiores tanto en el promedio, en las notas mínimas y en las notas máximas.

En cuanto a los resultados cualitativos se observa tanto en las opiniones seleccionadas de los estudiantes (que se muestran en la tabla 4 como en el resto de opiniones, que la metodología tradicional de clase sigue teniendo su audiencia sin embargo el uso intensivo de los recursos tecnológicos modernos, tanto en hardware como en software, son profundamente cautivadores para los alumnos pues se sintonizan con el docente cuando ven que éste hace uso del lenguaje natural de los jóvenes de hoy como son esas mismas expresiones tecnológicas. Una de las ventajas de recibir una clase de programación de computadores desplegando los programas a través del videobeam sobre una superficie plana es que los alumnos visualizan lo que sucede en codificación, corrección de errores, compilación y ejecución desde un escenario que es común para todos lo cual, en algún sentido, democratiza la sesión.

La tabla 5 resume las opiniones de los estudiantes y se puede observar en ella que son mayoritarias las opiniones favorables en referencia con la aplicación de la metodología sugerida que aquellas opiniones que se derivan de la ausencia de la metodología sugerida. En el I semestre del 2015 el 50\% de las opiniones fueron favorables en relación con la ausencia de la metodología y en ese mismo grupo casi en su totalidad $(90 \%)$ brindaron una opinión favorable en referencia con las sesiones en donde la metodología se aplicó. Para el II semestre del 2015 la relación porcentual fue similar, las opiniones favorables de la ausencia de la metodología sugerida fueron menores (45\%) que las opiniones favorables de la aplicación de dicha metodología (95\%) lo cual, según la opinión de los mismos estudiantes valida la propuesta metodológica que inspira este artículo.

\section{CONCLUSIONES}

- Es posible fortalecer la exposición magistral y el proceso de aprendizaje a partir de la utilización, apropiación, aplicación de las TIC tanto en su expresión de hardware como en sus facilidades de software

- Cuando se desarrollan procesos investigativos siempre resulta ser útil establecer parámetros que permitan confrontar las metodologías propuestas con las tradicionales y abrir espacios para que los estudiantes, protagonistas de la escena académica, conceptúen y opinen al respecto

- Conviene, en este tipo de investigaciones, asegurarse de que los temas seleccionados son cercanos, similares en contenido y en codificación (tratándose de programación de computadores) y que se imparten en las mismas condiciones endógenas y exógenas en relación con el aula de clases 
- Esta metodología se puede nutrir con el uso de más programas y recursos, enmarcados dentro de las TIC, dependiendo de las necesidades, gustos y preferencias que, a criterio del docente, convengan para un desarrollo apropiado de sus clases

- Es muy importante tener claro no tanto el uso, concepto y descripción de las TIC (tanto en hardware como en software) sino el uso que se le dé en el desarrollo de una sesión de clase porque es allí en donde estos recursos cobran la relevancia que pueden tener. De aquí se deduce que es posible que una clase con metodología tradicional sea más enriquecedora que aquella en donde se tengan muchos recursos pero que no se aprovechen apropiadamente

- Siempre conviene establecer parámetros cuantitativos que evalúen el conocimiento y permitan realizar comparaciones entre las diferentes metodologias utilizadas

- La opinión abierta de los estudiantes es muy importante y para ello han de posibilitarse los espacios apropiados debido a que son los que justifican el refinamiento de los procesos de aprendizaje y la búsqueda de metodologías que los potencialicen

- El análisis cuantitativo y el análisis cualitativo favorecen mucho la evaluación de este tipo de procesos dado que, al complementarse, brindan un panorama más objetivo que permite hacer una aproximación más clara a los resultados de este tipo de investigaciones

\section{BIBLIOGRAFÍA}

Ausubel, D. (1963). Psychology of meaningful verbal learning: an introduction to school learning. New York: Grune \& Straton.

Barriga Arceo, F., \& Hernandez Rojas, G. (2002). Estrategias docentes para un aprendizaje significativo: una interpretación constructivista. Ciudad de México: McGraw Hill Interamericana.

Briones, G. (1996). Metodología de la investigación cuantitativa en ciencias sociales. Bogotá: Instituto Colombiano para el Fomento de la Educación Superior.

Cortés, M., \& Iglesias, M. (2004). Generalidades sobre metodología de la investigación. Ciudad del Carmen, Campeche. México: Universidad Autónoma del Carmen.

Deitel \& Deitel. (2013). C++ Programming. New York: Prentice Hall.

Felleisen, M. e. (2006). How to design Programs. Boston: MIT Press.

Hall, S., Waitz, I., Brodeur, D., Soderholm, D., \& Nasr, R. (November de 2002). Adoption of Active Learning in a Lecture based Engineering Class. 32nd ASEE/IEEE Frontiers in Education Conference, Session T2A.

Hernandez Sampieri, R., Fernandez Collado, C., \& Baptista Lucio, P. (2006). Metodología de la Investigación (4a Edición ed.). Ciudad de México: McGraw Hillo Interamericana. 
LÍNEA DE INVESTIGACIÓN: DESARROLLO DE UN MODELO PEDAGÓGICO BASADO EN E-LEARNING

Lerma Gonzalez, H. (2014). Metodología de la Investigación: propuesta, anteproyecto y proyecto. Bogotá: ECOE ediciones.

Ministerio de las TIC, R. (2012). La formación de docentes en TIC. Bogotá: ExpreCards CI SAS.

Onrubia, J. (Febrero 2005). Aprender y enseñar en entornos virtuales. Revista de Educación a Distancia, 22-26.

Palomar Sanchez, M. (2009). Ventajas e inconvenientes de las TIC en la docencia. CSIF Revista, $1-8$.

Sabino, C. (2002). El proceso de investigación. Buenos Aires: Editorial Lumen.

Salinas, J. (Noviembre de 2004). Innovación docente y uso de las TIC en la enseñanza universitaria. Revista Universidad y Sociedad del Conocimiento, 1(1), 1-16.

Schildt, H. (2010). C Programming. México: McGraw Hill.

Schildt, H. (2010). C++ Programming. Vancouver: McGraw Hill.

Trejos Buriticá, O. (2000). La Esencia de la Lógica de Programación. Manizales: Centro Editorial Universidad de Caldas.

Trejos Buriticá, O. (2013). Significado y Competencias. Pereira: Papiro.

UNESCO. (Enero de 2008). Estándares de competencia en TIC para docentes. (UNESCO, Ed.) Recuperado el Junio de 2016, de http://www.eduteka.org/EstandaresDocentesUnesco.php

Van Roy, P. (2008). Techniques and methods in programming computer. Louvaine: University Press. 